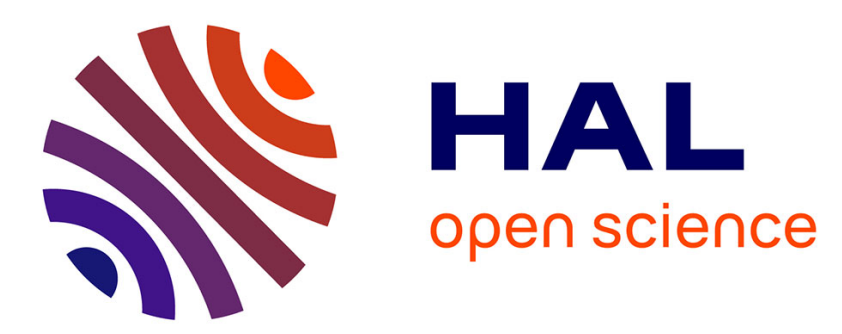

\title{
Blind Signal Detection Under Synchronization Errors for FSO Links with High Mobility
}

\author{
Mohammad Taghi Dabiri, Seyed Mohammad Sajad Sadough, Mohammad Ali
} Khalighi

\section{- To cite this version:}

Mohammad Taghi Dabiri, Seyed Mohammad Sajad Sadough, Mohammad Ali Khalighi. Blind Signal Detection Under Synchronization Errors for FSO Links with High Mobility. IEEE Transactions on Communications, 2019, 67 (10), pp.7006-7015. 10.1109/TCOMM.2019.2930254 • hal-02421401

\section{HAL Id: hal-02421401 https://hal.science/hal-02421401}

Submitted on 5 Apr 2020

HAL is a multi-disciplinary open access archive for the deposit and dissemination of scientific research documents, whether they are published or not. The documents may come from teaching and research institutions in France or abroad, or from public or private research centers.
L'archive ouverte pluridisciplinaire HAL, est destinée au dépôt et à la diffusion de documents scientifiques de niveau recherche, publiés ou non, émanant des établissements d'enseignement et de recherche français ou étrangers, des laboratoires publics ou privés. 


\title{
Blind Signal Detection Under Synchronization Errors for FSO Links with High Mobility
}

\author{
M. T. Dabiri, S. M. S. Sadough, M. A. Khalighi
}

\begin{abstract}
We consider the use of free-space optical communication for fast moving platforms such as high-speed trains where the sampling clock offset is randomly changing and, in addition, the receiver does not have any information on the instantaneous channel fading coefficient. By employing multiple samplers at the receiver, we propose a class of sequence detection methods for the case of OOK signaling without using any training sequence. First, we study maximum likelihood based detection, which has a relatively high computational complexity. Second, by employing generalized likelihood ratio test, we propose a more practical blind sequence detection method of reduced complexity. To further reduce the computational complexity, third, we propose a novel scheme that uses two wavelengths at the transmitter and differential blind detection at the receiver. Fourth, to benefit from diversity gain with this differential scheme, we consider the use of sufficiently different wavelengths, where we propose an efficient blind detection method. The pros and cons of each proposed detection method are contrasted through numerical results and their processing load are compared as well.
\end{abstract}

Index Terms-Free-space optics; blind data detection; differential signaling; synchronization; atmospheric turbulence.

\section{INTRODUCTION}

Free-space optical (FSO) communication is well known as a promising wireless access technology thanks to its features of huge available license-free spectrum, low-cost implementation, and robustness to electromagnetic interference [2]. Due to the relatively low implementation complexity, intensity modulation with direct detection (IM/DD) based on On-Off keying (OOK) is used in most current commercial FSO systems. Nevertheless, for optimal signal demodulation, adaptive threshold setting is required at the receiver ( $\mathrm{Rx})$, which needs, in turn, accurate channel estimation [3]. Channel estimation is classically done through the transmission of multiple pilot symbols per frame. However, this pilot overhead results in a spectral efficiency loss, in particular for the case of fast-varying channels [4], [5]. To avoid transmitting pilot symbols, a few earlier works have investigated maximumlikelihood (ML) sequence detection and its generalization [6], [7]. However, the proposed methods generally suffer from high computational complexity which may be of critical concern for practical implementation of FSO systems. Therefore, some more recent works, such as [8]-[16], have tried to design

M. T. Dabiri and S. M. S. Sadough are with the Department of Electrical Engineering, Shahid Beheshti University G. C., 1983969411, Tehran, Iran (Email: \{m_dabiri, s_sadough\}@ sbu.ac.ir).

M.A. Khalighi is with Aix-Marseille University, CNRS, Centrale Marseille, Institut Fresnel, Marseille, France (Email: ali.khalighi@fresnel.fr).

Parts of this work have been presented at the 11th International Symposium on Communication Systems, Networks, and Digital Signal Processing (CSNDSP), Budapest, Hungary, July 2018, Reference [1]. lower complexity signal detection methods. However, in [8][16] it i assumed that the transceiver is perfectly synchronized. Although, such assumption is a valid for the fixed FSO links, this is not the case for mobile FSO link scenarios.

\section{A. FSO for high-mobility platforms}

In this paper we consider the use of the FSO technology for providing high data-rate wireless access for high-speed trains, which has attracted a great deal of attention in the past few years. Indeed, the demand of passengers to access broadband services with high quality in high-speed trains is exponentially increasing. Current radio-frequency wireless technologies such as the 4G (LTE, Long Term Evolution) and 4G+ (LTE-Advanced) networks can offer unreliable handovers and limited data rate, resulting in unsatisfying quality-ofexperience for users [17], [18]. Within this context, FSO has been recognized as a promising connectivity solution [18]-[20]. However, apart from the requirement of highly accurate and agile beam tracking, one of the most important challenges in such systems is time synchronization between the transmitter (Tx) and the Rx. This is, of course, in addition to the need to channel estimation, explained above. In fact, in most related literature, OOK modulation is considered assuming signal detection under the assumption of perfectly synchronized (PSY) Tx and Rx, which is practically not valid for FSO links with high mobility. To clarify better this point, consider a train with speed of $360 \mathrm{Km} / \mathrm{h}$ or $100 \mathrm{~m} / \mathrm{s}$. Given the speed of light $c=3 \times 10^{8} \mathrm{~m} / \mathrm{s}$, only after $1 \mathrm{~ms}$, the propagation delay of considered ground-to-train link changes equal to $\Delta_{\tau_{p}}=\frac{100 \mathrm{~m} / \mathrm{s} \times 1 \mathrm{~ms}}{3 \times 10^{8} \mathrm{~m} / \mathrm{s}}=0.33 \times 10^{-9}$. Now, consider a ground-to-train FSO link with a bit-rate of $R_{b}=1 \mathrm{Gbps}$. For OOK modulation, $R_{b}=1 \mathrm{Gbps}$ is obtained when bit duration $T_{b}=1 / R_{b}=1 \mathrm{~ns}$. Hence, only after $1 \mathrm{~ms}$, the sampling time at the $\mathrm{Rx}$ is deviated by at least $\tau=\Delta_{\tau_{p}}=$ $0.33 \times 10^{-9}=0.33 T_{b}$. This can result in a significant link performance degradation. Moreover, due to the Doppler effect, the coherence time of the underlying channel is reduced significantly. Hence, for reliable communication we would need to adjust the synchronization and the channel estimate at intervals much shorter than $1 \mathrm{~ms}$, which requires the insertion of several pilot bits inside each data frame. Obviously, this results in a considerable spectral efficiency loss and increased system complexity. These considerations suggest the practical interest of blind signal detection for such FSO links.

\section{B. Contributions}

In this work, we assume that the sampling clock offset is randomly changing and, in addition, the $\mathrm{Rx}$ does not have 
access to the instantaneous channel fading coefficient. First, by using $N_{s}$ samplers at the $\mathrm{Rx}$, we propose a blind sequence detection method over an observation window of length $L$. This method, which relies on the knowledge of the distribution of the channel attenuation, can achieve a performance close to the Rx with PSY and perfect channel state information (PCSI).

Second, based on the generalized likelihood ratio test (GLRT) criterion, we propose a suboptimal and more practical blind sequence detection method. Despite the need to larger values of $L$ to achieve performance comparable to the ideal $\mathrm{Rx}$, the proposed method can significantly reduce the computational complexity of conventional ML-based method.

Third, by employing differential OOK signaling with the expense of using two optical sources (emitting on two different wavelengths) and two photo-detectors, we propose a more efficient blind data detection scheme. Differential signaling is an innovative method that was firsty introduced in [21] to suppress the effect of background noise, and later proposed in [22], [23] to resolve the problem of detection threshold adjustment for OOK demodulation. It was also used in [24] to mitigate pointing errors effects, and in [25] to explore a onebit channel information scheme for a $2 \times 1$ FSO link. When the two wavelengths are sufficiently different, differential signaling can benefit from a diversity gain against channel fading [25]. As the fourth contribution, we propose an efficient blind detection scheme for such a scheme, albeit accepting an increased detection complexity.

The performances of the proposed methods are investigated through numerical results. We also compare the processing load of the four proposed detection methods.

\section{Paper structure}

The reminder of this paper is organized as follows. In Section II, we describe our system model and main assumptions. Our proposed signal detection schemes for the case of using a single wavelength are described in Section III along with the related simulation results and analyses. Next, in Section IV, we propose blind signal detection methods under differential signaling, and investigate their performance. Lastly, Section V concludes the paper.

\section{SySTEM MODEL}

As mentioned in the introduction, we consider an IM/DD FSO link using OOK modulation. As explained, due to high mobility of the Tx/Rx and high data rate, the sampling clock offset $\tau$ is randomly changing. At the $\mathrm{Rx}$, we use $N_{s}$ samplers that we denote by $\mathrm{SP}_{\mathrm{i}}, i \in\left\{1,2, \ldots, N_{s}\right\}$, see from Fig. 1 for $N_{s}=3$. Let $\tau$ be the sampling clock offset between the Tx and $\mathrm{SP}_{1}$. We assume that $\tau$ is an unknown parameter and has a uniform distribution in $\left(-T_{b}, T_{b}\right)$. As shown in Fig. $1, \mathrm{SP}_{\mathrm{i}}$ samples the $i$-th integrator output at time instants $t=\tau_{i}^{\prime}+k T_{b}$, where $\tau_{i}^{\prime}=\tau+(i-1) T_{b} / N_{s}$ with $k$ denoting the $k$-th bit interval. Prior to each sampler, an optical delay-line is inserted to apply a time shift of $(i-1) T_{b} / N_{s}$, for the $i$-th sampler. In practice, precise and stable adjustment of the amount of the delay is possible using small-size fiber-based delay-lines. ${ }^{1}$

\section{A. Signal model for a simple link using a single wavelength}

We consider here the use of an APD at the Rx. As explained and as depicted in Fig. 2, due to the sampling clock offset $\tau$, at the $\mathrm{Rx},\left(1-\tau_{i} / T_{b}\right)$ of $\left(k+k_{i}^{\prime}\right)$ th transmitted signal along with the $\tau_{i} / T_{b}$ of $\left(k+k_{i}^{\prime}+1\right)$ th transmitted signal are placed at the $k$ th pulse duration interval of receiver. Hence, for OOK modulation, the photo-current output of $\mathrm{SP}_{\mathrm{i}}$ corresponding to the $k$-th bit interval can be written as

$$
r_{k}^{\prime(i)}=\eta h\left(1-\frac{\tau_{i}}{T_{b}}\right) s_{k+k_{i}^{\prime}}+\eta h \frac{\tau_{i}}{T_{b}} s_{k+k_{i}^{\prime}+1}+n_{k+k_{i}^{\prime}}^{(i)},
$$

where $\tau_{i}=\tau_{i}^{\prime}-k_{i}^{\prime} T_{b}, \tau_{i} \in\left\{0, T_{b}\right\} ; k_{i}^{\prime}=\left\lfloor\tau_{i}^{\prime} / T_{b}\right\rfloor$ with $\lfloor z\rfloor$ being the floor function; $h$ denotes the instantaneous channel coefficient assumed to be constant over a large number of transmitted bits; and $s_{k} \in\{0,1\}$ denotes the transmitted OOK symbols. Also, $\eta=2 \xi P_{t}$, where $P_{t}$ is the average transmitted power, $\xi=e G \mu / \tilde{h}_{p} \nu, G$ is the APD gain, $e$ denotes the electron charge, $\mu$ is the APD quantum efficiency, $\nu$ is the optical frequency, and $\tilde{h}_{p}$ stands for the Planck constant. Lastly, $n_{k}^{(i)}$ is the zero mean Gaussian noise with variance $\sigma_{n, k}^{2}$ that includes thermal and shot noises, and can be accurately modeled by a Gaussian distribution [26]. We have:

$$
\sigma_{n, k}^{2}=\sigma_{s}^{2} h s_{k}^{\prime \prime}+\sigma_{0}^{2},
$$

where $s_{k}^{\prime \prime}=\left(1-\tau_{i} / T_{b}\right) s_{k}+\tau_{i} s_{k+1}$, and $\sigma_{s}^{2}$ and $\sigma_{0}^{2}$ denote the variances of shot noise and signal-independent (thermal and background) noises, respectively. This model can be simplified to the case of a PIN photo-detector by setting $G=1$ and $\sigma_{s}^{2} \approx 0$ [26]. According to (1), the output of the sampler $\mathrm{SP}_{\mathrm{i}}$ at the time sample $k$ depends on the transmitted signals at time samples $k+k_{i}^{\prime}$ and $k+k_{i}^{\prime}+1$. In this paper, we assume that $k_{i}^{\prime}$ is perfectly known at the Rx. ${ }^{2}$ Let us define $r_{k}^{(i)}$ as $r_{k}^{(i)}=r_{k-k_{i}^{\prime}}^{\prime(i)}$. For simplicity and without loss of generality, in the sequel, we assume $T_{b}=1$. Accordingly, see Figs. 1 and 2,

$$
r_{k}^{(i)}=\eta h\left(1-\tau_{i}\right) s_{k}+\eta h \tau_{i} s_{k+1}+n_{k}^{(i)} .
$$

For signal detection, we should reasonably use the output of the sampler (say, the $i$-th), for which $0 \leq \tau_{i}<1 / N_{s}$. More precisely, we have:

$$
\begin{aligned}
\tau_{i} & =\tau+(i-1) / N_{s}-k_{i}^{\prime} \\
& =\tau+(i-1) / N_{s}-\left\lfloor\tau+(i-1) / N_{s}\right\rfloor .
\end{aligned}
$$

From (4) and as shown in Fig. 2, for any given $\tau$, only one of the $\tau_{i}$ s falls in the interval $\left[0,1 / N_{s}\right)$. This allows us to simplify the detector's structure, as we will see in the next section. For instance, for $N_{s}=5$, we are sure that the synchronization error between transmitter and the considered $i$ th sampler is

\footnotetext{
${ }^{1}$ Such a fiber-based optical delay-line consists of an input and output fiber collimator to project the light into free space and collect it back into the fiber. The distance the light travels (and hence, the delay) can be accurately controlled, e.g., by fixing the separation between the input and output optics.

${ }^{2}$ Note that $k_{i}^{\prime}$ only takes three values of $\{-1,0,1\}$, depending on $\tau$. Also, it changes much slower than $\tau$, which makes it possible to estimate it with high accuracy. The estimation of $k_{i}^{\prime}$ is the beyond the scope of this paper and we assume it is perfectly known at the Rx.
} 


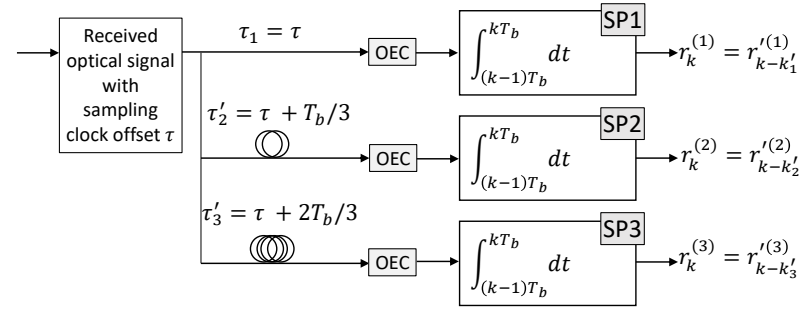

Fig. 1. Block diagram of sampler for $N_{s}=3$. OEC stands for optical to electrical conversion.

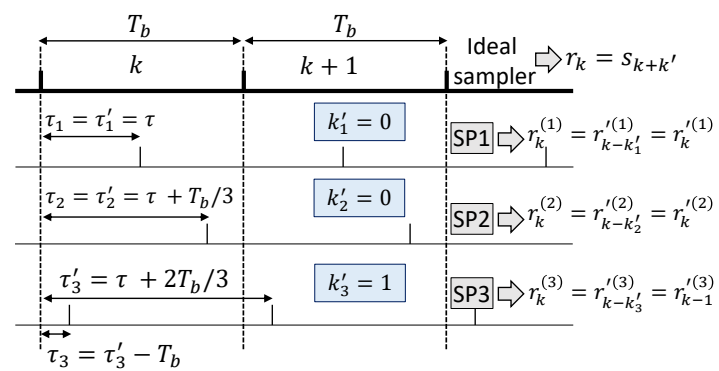

(a)

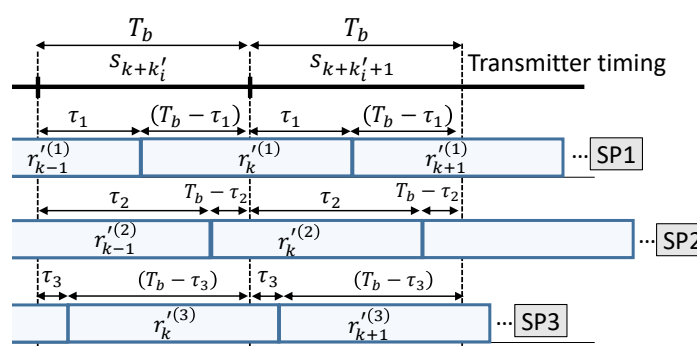

(b)

Fig. 2. Illustration of samplers' outputs corresponding to Fig. 1 for the case where the offset clock between the Tx and the first sampler $\left(\mathrm{SP}_{1}\right)$ is $\tau$ : (a) showing the relationship between $\tau_{i}$ and $\tau_{i}^{\prime}$, and also $r_{k}^{(i)}$ and $r_{k}^{(i)}$; (b) showing the relationship between $r_{k}^{\prime(i)}$ and $s_{k}$.

lower than 0.2 , or $0 \leq \tau_{i}<0.2$ and by increasing $N_{s}$, we expect the synchronization error decreases. Hence, for data detection, we first estimate $i$ for which $\tau_{i} \in\left[0,1 / N_{s}\right)$. Then, we use the outputs of this sampler (which changes over time due to link mobility) for data detection.

Finally, from (3) and due to the one-to-one relationship between $\tau$ and $\tau_{i}$, we have

$$
\begin{aligned}
p\left[r_{k}^{(i)} \mid h\right. & \left., \tau, s_{k}, s_{k+1}\right]=p\left[r_{k}^{(i)} \mid h, \tau_{i}, s_{k}, s_{k+1}\right] \\
& =\frac{1}{\sqrt{2 \pi\left(h\left(\left(1-\tau_{i}\right) s_{k}+\tau_{i} s_{k+1}\right) \sigma_{s}^{2}+\sigma_{0}^{2}\right)}} \\
& \times \exp \left(-\frac{\left|r_{k}^{(i)}-\eta h\left(1-\tau_{i}\right) s_{k}-\eta h \tau_{i} s_{k+1}\right|^{2}}{2 h\left(\left(1-\tau_{i}\right) s_{k}+\tau_{i} s_{k+1}\right) \sigma_{s}^{2}+2 \sigma_{0}^{2}}\right) .
\end{aligned}
$$

\section{B. Signal Model for OOK with Differential Signaling}

By differential signaling, at the $\mathrm{Tx}$, at time sample $k$, the OOK signal $s_{k}$ and its complement $\bar{s}_{k}=1-s_{k}$ are used to drive two distinct optical sources (OSs) working on

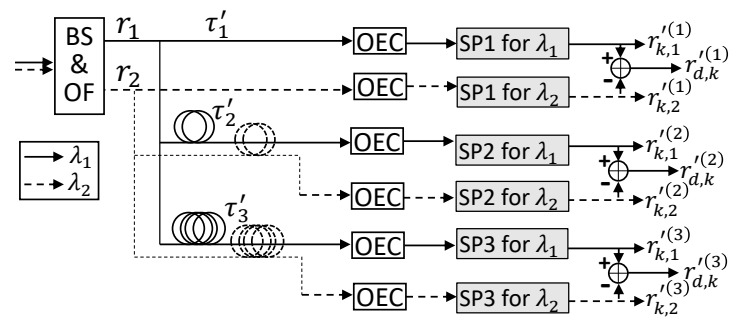

Fig. 3. Proposed block diagram of sampler for the case of differential signaling.

wavelengths $\lambda_{1}$ and $\lambda_{2}$ [21]. The outputs of the OSs are passed through a beam combiner (BC) before sending over the FSO channel. To keep the same average transmitted power of $P_{t}$ as in the previous case, we set $\eta=\xi P_{t}$.

At the $\mathrm{Rx}$, the optical signal is passed through a beam splitter (BS) whose outputs are fed into two optical filters (OFs) with center wavelengths on $\lambda_{1}$ and $\lambda_{2}$ in order to separate the received optical signals from the two lasers. Then, after optical-to-electrical conversion (OEC), signals $r_{1}$ and $r_{2}$ are time-sampled to get $r_{k, 1}^{\prime(i)}$ and $r_{k, 2}^{\prime(i)}$, respectively. Then, $r_{k, 2}^{(i)}$ is subtracted from $r_{k, 1}^{\prime(i)}$ to get $r_{d, k}^{\prime(i)}$ (see Fig. 3).

Let $h_{1}$ and $h_{2}$ be the channel fading coefficients corresponding to $\lambda_{1}$ and $\lambda_{2}$, respectively. Similar to the formulation of $r_{k}^{(i)}=r_{k-k_{i}^{\prime}}^{(i)}$, here $r_{k, 1}^{(i)}=r_{k-k_{i}^{\prime}, 1}^{\prime(i)}, r_{k, 2}^{(i)}=r_{k-k_{i}^{\prime}, 2}^{(i)}$ and $r_{d, k}^{(i)}=r_{d, k-k_{i}^{\prime}}^{\prime(i)}$. Hence, the photo-current outputs of $\mathrm{SP}_{\mathrm{i}}$ corresponding to the $k$-th bit interval are given by:

$$
\left\{\begin{aligned}
r_{d, k}^{(i)} & =r_{k, 1}^{(i)}-r_{k, 2}^{(i)} \\
r_{k, 1}^{(i)} & =\eta\left(1-\tau_{i}\right) h_{1} s_{k}+\eta \tau_{i} h_{1} s_{k+1}+n_{k, 1}^{(i)} \\
r_{k, 2}^{(i)} & =\eta\left(1-\tau_{i}\right) h_{2} \bar{s}_{k}+\eta \tau_{i} h_{2} \bar{s}_{k+1}+n_{k, 2}^{(i)}
\end{aligned}\right.
$$

where $n_{k, 1}^{(i)}$ and $n_{k, 2}^{(i)}$ are zero-mean Gaussian noises with variances:

$$
\left\{\begin{array}{l}
\sigma_{n, 1}^{2}=\sigma_{s}^{2} h_{1} s_{k}^{\prime \prime}+\sigma_{0}^{2} \\
\sigma_{n, 2}^{2}=\sigma_{s}^{2} h_{2} \bar{s}_{k}^{\prime \prime}+\sigma_{0}^{2},
\end{array}\right.
$$

and

$$
\bar{s}_{k}^{\prime \prime}=\left(1-\tau_{i}\right) \bar{s}_{k}+\tau_{i} \bar{s}_{k+1} .
$$

Also, the variance of $n_{d, k}^{(i)}=n_{k, 1}^{(i)}-n_{k, 2}^{(i)}$ is:

$$
\sigma_{d, n}^{2}=\sigma_{s}^{2}\left(h_{1} s_{k}^{\prime \prime}+h_{2} \bar{s}_{k}^{\prime \prime}\right)+2 \sigma_{0}^{2} .
$$

For the case of close $\lambda_{1}$ and $\lambda_{2}$, we have $h_{1} \approx h_{2}$ [21]. Otherwise, when these wavelengths are sufficiently apart, we can assume that $h_{1}$ and $h_{2}$ are almost independent.

\section{Channel Model}

In our channel model, we consider the joint effect of deterministic propagation loss, denoted by $h_{a}$, and the random intensity fluctuation due to atmospheric turbulence, denoted by $h_{l}$, while neglecting the effect of pointing errors. In fact, in order to compensate pointing errors, recent FSO systems employ efficient tracking mechanisms based on electro-optic 
or acousto-optic devices [27], [28], which is particularly applicable to the case of fast moving platforms [29]. In the sequel, we do not consider the effect of pointing errors by assuming that an efficient automatic beam pointing, acquisition and tracking system is employed [30]. The channel attenuation is then considered as $h=h_{l} h_{a}$. To model $h_{a}$ we use the gamma-gamma (G-G) distribution that is accepted as a general model describing all turbulence regimes, with the following probability density function (PDF) [31]:

$$
f_{G G}\left(h_{a}\right)=\frac{2(\alpha \beta)^{\frac{\alpha+\beta}{2}}}{\Gamma(\alpha) \Gamma(\beta)} h_{a}^{\frac{\alpha+\beta}{2}-1} k_{\alpha-\beta}\left(2 \sqrt{\alpha \beta h_{a}}\right) .
$$

where, $\Gamma($.$) is the gamma function, k_{m}($.$) is the modified$ Bessel function of order $m$ and second kind, and parameters $\alpha$ and $\beta$ are related to turbulence conditions.

\section{Signal Detection for Single-WaVelength TRANSMISSION}

Under PCSI and PSY at the Rx, given the received signal $r_{k}=\eta h s_{k}+n_{k}$, the transmitted signal $s_{k} \in\{0,1\}$ can be detected easily as: $r_{k} \stackrel{\hat{s}_{k}=1}{\gtrless} \frac{\eta h}{2}$. In general sense, blind signal detection may involve ambiguity on the instantaneous channel fading coefficient and synchronization (i.e., the correct sampling time). In the proposed blind methods, we avoid ambiguity on the instantaneous channel fading coefficient by exploiting the slow fading property of the FSO channel through sequence symbol detection. In addition, we avoid ambiguity in the synchronization by using $N_{s}$ samplers.

We assume here that we do not have any information on $\tau$ or $h$; our goal being to detect OOK signals without requiring any pilot symbol. We propose two detection methods for this purpose. The first method detects the sequence of OOK signals under the assumption that the Rx knows the channel fading distribution $f_{h}(h)$, whereas the second proposed method does not require this PDF. It is worth mentioning that although for a static FSO link the channel distribution parameters typically change very slowly over time and we do not estimate them frequently, in the high mobility context that we consider in this paper, their estimation may be an important issue.

For sequence data detection, we arrange the outputs of all samplers in "observation windows" composed of $L$ intervals. Let us denote by $\mathbf{r}_{i}(L)=\left\{r_{1}^{(i)}, r_{2}^{(i)}, \ldots, r_{L}^{(i)}\right\}$ the vector of outputs of the sampler $\mathrm{SP}_{\mathrm{i}}$ corresponding to the transmitted signals $\mathbf{s}(L+1)=\left\{s_{1}, s_{2}, \ldots, s_{L+1}\right\} .{ }^{3}$ Based on (2) and (3), the PDF of $\mathbf{r}_{i}(L)$ conditioned on $h, \tau_{i}$, and $\mathbf{s}(L+1)$ is:

$$
p\left[\mathbf{r}_{i}(L) \mid h, \tau_{i}, \mathbf{s}(L+1)\right]=\prod_{k=1}^{L} p\left[r_{k}^{(i)} \mid h, \tau_{i}, s_{k}, s_{k+1}\right],
$$

where $p\left[r_{k}^{(i)} \mid h, \tau_{i}, s_{k}, s_{k+1}\right]$ is given in (5).

\footnotetext{
${ }^{3}$ Note that, from (3), a received sequence with length $L$ includes the contribution of $L+1$ transmitted symbols. Therefore, we limit the samplers' outputs to $L$ samples.
}

\section{A. Optimal ML-based Sequence Detection Method}

Based on ML criterion, signal detection is performed as:

$$
\hat{\mathbf{s}}(L)=\underset{\mathbf{s}(L+1)}{\arg \max } \mathcal{M}_{\mathrm{ML}}(\mathbf{s}(L+1)),
$$

where

$$
\mathcal{M}_{\mathrm{ML}}(\mathbf{s}(L+1))=\int_{0}^{\infty} \mathcal{M}_{\mathrm{ML}}^{\prime}(\tau) f_{h}(h) d h,
$$

and

$$
\begin{aligned}
\mathcal{M}_{\mathrm{ML}}^{\prime}(\tau) & =\int \prod_{0}^{1} \prod_{i=1}^{N_{s}} p\left[\mathbf{r}_{i}(L) \mid h, \tau, \mathbf{s}(L+1)\right] d \tau, \\
& =\int \prod_{0}^{1} \prod_{i=1}^{N_{s}} \prod_{k=1}^{L} p\left[r_{k}^{(i)} \mid h, \tau, s_{k}, s_{k+1}\right] d \tau .
\end{aligned}
$$

Given (5), finding a general closed-form expression for the integral in (14) is quite difficult.

As we can see, the proposed method detects $\hat{\mathbf{s}}(L)$ by searching over $2^{L+1}$ possible states of the metric (13), each one involving the calculation of a two-dimensional integral. In addition, this method requires the knowledge of the parameters $\alpha$ and $\beta$ of the channel PDF $f_{h}(h)$. A simple method for estimating the parameters of a G-G PDF was recently proposed in [32]. This method relies on the estimation of the moments of $h$ of orders $k,(k+1), l$, and $(l+1)$, that should be calculated over a sufficiently large sample size.

Overall, as explained above, this method suffers from relatively high computational complexity for a practical and realtime implementation.

\section{B. Suboptimal GLRT-based Sequence Detection Method}

We propose here a sub-optimal detector based on the GLRT criterion, which has a lower computational complexity and does not require the channel PDF. From [33], [34], the GLRT method jointly decides on $h$ and $\mathbf{s}(L)=\left\{s_{1}, \ldots, s_{L}\right\}$ as:

$$
\begin{aligned}
\hat{h}, \hat{\mathbf{s}}(L) & =\underset{h, \mathbf{s}(L+1)}{\arg \max } \prod_{i=1}^{N_{s}} p\left[\mathbf{r}_{i}(L) \mid h, \mathbf{s}(L+1)\right] \\
& =\underset{h, \mathbf{s}(L+1)}{\arg \max } \int \prod_{i=1}^{N_{s}} p\left[\mathbf{r}_{i}(L) \mid h, \tau, \mathbf{s}(L+1)\right] d \tau .
\end{aligned}
$$

To reduce the computational complexity of (15), we propose a detection method which is inspired by GLRT that we refer to it as GLRT-based detection method. This detector jointly decides on $h, i$, and $\mathbf{s}(L)=\left\{s_{1}, \ldots, s_{L}\right\}$ :

$$
\hat{i}, \hat{h}, \hat{\mathbf{s}}(L)=\underset{i, h, \mathbf{s}(L+1)}{\arg \max } p\left[\mathbf{r}_{i}(L) \mid h, \mathbf{s}(L+1)\right] .
$$

When $\tau_{i}$ is known, we have $p\left[\mathbf{r}_{i}(L) \mid h, \mathbf{s}(L+1)\right]=$ $p\left[\mathbf{r}_{i}(L) \mid \tau_{i}, h, \mathbf{s}(L+1)\right]$. As mentioned previously, one $\tau_{i}$, corresponding to an $i$ th min, $i \in\left\{1, \ldots, N_{s}\right\}$, falls in the interval $\left[0,1 / N_{s}\right)$. For this special $\tau_{i}$, the mean and the variance are equal to $\bar{\tau}_{i}=1 / 2 N_{s}$ and $\sigma_{\tau_{i}}^{2}=T_{b}^{2} / 12 N_{s}^{2}=$ $1 / 12 N_{s}^{2}$, respectively. Given that $\sigma_{\tau_{i}}^{2}$ decreases by increasing 
$N_{s}$, assuming sufficiently large $N_{s}$, we estimate $\tau_{i}$ by its mean $\bar{\tau}_{i}$. Then, we can simplify (16) as

$$
\hat{i}, \hat{h}, \hat{\mathbf{s}}(L)=\underset{i, h, \mathbf{s}(L+1)}{\arg \max } p\left[\mathbf{r}_{i}(L) \mid \tau_{i}=\frac{1}{2 N_{s}}, h, \mathbf{s}(L+1)\right] .
$$

Equivalently,

$$
\begin{aligned}
& \hat{i}, \hat{h}, \hat{\mathbf{s}}(L)=\underset{i, h, \mathbf{s}(L+1)}{\arg \max } \log p\left[\mathbf{r}_{i}(L) \mid \tau_{i}=\frac{1}{2 N_{s}}, h, \mathbf{s}(L+1)\right] \\
& \quad=\underset{i, h, \mathbf{s}(L+1)}{\arg \min } \sum_{k=1}^{L} \frac{\left|r_{k}^{(i)}-\eta h\left(1-\frac{1}{2 N_{s}}\right) s_{k}-\frac{\eta h}{2 N_{s}} s_{k+1}\right|^{2}}{h\left(\left(1-\frac{1}{2 N_{s}}\right) s_{k}+\frac{1}{2 N_{s}} s_{k+1}\right) \sigma_{s}^{2}+\sigma_{0}^{2}} \\
& \quad+\log \left(h\left(\left(1-\frac{1}{2 N_{s}}\right) s_{k}+\frac{1}{2 N_{s}} s_{k+1}\right) \sigma_{s}^{2}+\sigma_{0}^{2}\right) .
\end{aligned}
$$

For notation simplicity, we define

$$
s_{k}^{\prime}=\left(1-\frac{1}{2 N_{s}}\right) s_{k}+\frac{1}{2 N_{s}} s_{k+1} .
$$

We have then,

$\hat{i}, \hat{h}, \hat{\mathbf{s}}(L)=\underset{i, h, \mathbf{s}(L+1)}{\arg \min } \sum_{k=1}^{L} \frac{\left|r_{k}^{(i)}-\eta h s_{k}^{\prime}\right|^{2}}{h s_{k}^{\prime} \sigma_{s}^{2}+\sigma_{0}^{2}}+\log \left(h s_{k}^{\prime} \sigma_{s}^{2}+\sigma_{0}^{2}\right)$

For the given $i$ and $\mathbf{s}(L+1)$, by differentiating (20) with respect to $h$ and setting it to zero, we obtain,

$$
\begin{aligned}
\hat{h}= & -\frac{\left(2 \eta^{2} \sigma_{0}^{2}+\sigma_{s}^{2}\right) \mathcal{S}_{2}^{\prime}}{2 \eta^{2} \sigma_{s}^{2} \mathcal{S}_{3}^{\prime}} \\
& +\left[\frac { 2 \sigma _ { 0 } ^ { 2 } } { \eta \sigma _ { s } ^ { 2 } \mathcal { S } _ { 3 } ^ { \prime } } \left(\left(1-\frac{1}{2 N_{s}}\right)\left(\mathcal{R}_{o n, 1}^{(i)}+r_{L}^{(i)} s_{L}\right)\right.\right. \\
& \left.+\frac{1}{2 N_{s}}\left(\mathcal{R}_{o n, 1}^{(i)}+r_{L}^{(i)} s_{L+1}\right)\right) \\
& +\frac{1}{\eta^{2} \mathcal{S}_{3}^{\prime}}\left(\left(1-\frac{1}{2 N_{s}}\right)\left(\mathcal{R}_{o n, 2}^{(i)}+\left(r_{L}^{(i)}\right)^{2} s_{L}\right)\right. \\
& \left.+\frac{1}{2 N_{s}}\left(\mathcal{R}_{o n, 2}^{\prime(i)}+\left(r_{L}^{(i)}\right)^{2} s_{L+1}\right)\right) \\
& +\frac{\left(2 \eta^{2} \sigma_{0}^{2}+\sigma_{s}^{2}\right)^{2}\left(\mathcal{S}_{2}^{\prime}\right)^{2}-2 \eta^{2} \sigma_{0}^{2} \sigma_{s}^{4} \mathcal{S}_{1}^{\prime} \mathcal{S}_{3}^{\prime}}{\left(2 \eta^{2} \sigma_{s}^{2} \mathcal{S}_{3}^{\prime}\right)^{2}},
\end{aligned}
$$

where

$$
\left\{\begin{array}{l}
\mathcal{S}_{1}^{\prime}=\sum_{k=1}^{L} s_{k}^{\prime}=\left(1-\frac{1}{2 N_{s}}\right) s_{1}+\frac{1}{2 N_{s}} s_{L+1}+N_{o n} \\
\mathcal{S}_{2}^{\prime}=\sum_{k=1}^{L} s_{k}^{\prime 2} \\
\mathcal{S}_{3}^{\prime}=\sum_{k=1}^{L} s_{k}^{\prime 3}
\end{array}\right.
$$

${ }^{4}$ We will see in the numerical results that $N_{s} \geq 4$ is large enough to provide a good Rx performance. and also, $N_{\text {on }}=\sum_{k=2}^{L} s_{k}$ is the number of bits ' 1 ' in the vector $\mathbf{s}^{\prime}(L-1)=\left\{s_{2}, s_{3}, \ldots, s_{L}\right\}$, and

$$
\left\{\begin{aligned}
\mathcal{R}_{\text {on }, 1}^{(i)} & =\sum_{k=1}^{L-1} r_{k}^{(i)} s_{k} \\
\mathcal{R}_{\text {on }, 1}^{\prime(i)} & =\sum_{k=1}^{L-1} r_{k}^{(i)} s_{k+1} \\
\mathcal{R}_{\text {on }, 2}^{(i)} & =\sum_{k=1}^{L-1}\left(r_{k}^{(i)}\right)^{2} s_{k} \\
\mathcal{R}_{\text {on }, 2}^{\prime(i)} & =\sum_{k=1}^{L-1}\left(r_{k}^{(i)}\right)^{2} s_{k+1} .
\end{aligned}\right.
$$

Now, the detection method in (20) is simplified as

$$
\hat{i}, \hat{\mathbf{s}}(L)=\underset{i, \mathbf{s}(L+1)}{\arg \min } \sum_{k=1}^{L} \frac{\left|r_{k}^{(i)}-\eta \hat{h} s_{k}^{\prime}\right|^{2}}{\hat{h} s_{k}^{\prime} \sigma_{s}^{2}+\sigma_{0}^{2}}+\log \left(\hat{h} s_{k}^{\prime} \sigma_{s}^{2}+\sigma_{0}^{2}\right),
$$

where $\hat{h}$ is calculated from (21). Note that for the case of an all-zero transmitted sequence, i.e., $\mathbf{s}(L+1)=0$, the proposed estimator in (21) does not work correctly. In such a case, from (19), we have $s_{k}^{\prime}=0, k=1, \ldots, L$, and consequently, we have $\mathcal{S}_{2}^{\prime}=0$ and $\mathcal{S}_{3}^{\prime}=0$ from (22). As a result, we can not use (21) to calculate $\hat{h}$. To resolve this issue, we propose an alternative to (24) as follows:

$$
\hat{i}, \hat{\mathbf{s}}(L)=\underset{i, \mathbf{s}(L+1)}{\arg \min }\left\{\begin{array}{l}
\mathcal{A}_{1} \text { for } \mathbf{s}(L+1) \neq 0 \\
\mathcal{A}_{0} \text { for } \mathbf{s}(L+1)=0
\end{array},\right.
$$

where

$$
\left\{\begin{array}{l}
\mathcal{A}_{1}=\sum_{k=1}^{L} \frac{\left|r_{k}^{(i)}-\eta \hat{h} s_{k}^{\prime}\right|^{2}}{\hat{h} s_{k}^{\prime} \sigma_{s}^{2}+\sigma_{0}^{2}}+\log \left(\hat{h} s_{k}^{\prime} \sigma_{s}^{2}+\sigma_{0}^{2}\right) \\
\mathcal{A}_{0}=L \log \left(\sigma_{0}^{2}\right)+\mathcal{R}_{\text {tot }, 2}^{(i)} / \sigma_{0}^{2}
\end{array}\right.
$$

and

$$
\mathcal{R}_{\text {tot }, 2}^{(i)}=\sum_{k=1}^{L}\left(r_{k}^{(i)}\right)^{2}
$$

Lastly, in the case of a thermal noise limited Rx, metrics of $\mathcal{A}_{1}$ and $\mathcal{A}_{0}$ are simplified as follows:

$$
\left\{\begin{aligned}
\mathcal{A}_{1}= & \mathcal{R}_{\text {tot }, 2}^{(i)}-\frac{\left(\left(1-\frac{1}{2 N_{s}}\right) \mathcal{R}_{\text {on, }}^{(i)}+\frac{1}{2 N_{s}} \mathcal{R}_{o n, 1}^{(i)}\right)^{2}}{\mathcal{S}_{2}^{\prime}} \\
& -\frac{\left(\left(1-\frac{1}{2 N_{s}}\right) r_{1}^{(i)} s_{1}+\frac{1}{2 N_{s}} r_{L}^{(i)} s_{L+1}\right)^{2}}{\mathcal{S}_{2}^{\prime}}, \\
\mathcal{A}_{0}= & \mathcal{R}_{\text {tot }, 2}^{(i)} .
\end{aligned}\right.
$$

Note that, unlike the metric of (13) which requires the calculation of a two-dimensional integral, the metric in (25),(26) only needs a series of simple additions and multiplications and also does not require the knowledge of $f_{h}(h)$. Here, the $\mathrm{Rx}$ searches among $N_{s} \times 2^{L+1}$ possible received sequences (i.e., $2^{L+1}$ possible bit sequences for each sampler output) to find the sequence that minimizes (25). To avoid this exhaustive search and to further reduce the $\mathrm{Rx}$ complexity, we have developed a fast search algorithm for implementing (25), which reduces the search space to $4 N_{s} L$ states. Details of this algorithm are presented in Appendix. 
TABLE I

SySTEM PARAMETERS FOR SimUlations

\begin{tabular}{lcc}
\hline \hline Description & Parameter & Setting \\
\hline \hline APD Gain & $G$ & 100 \\
APD Quantum Efficiency & $\mu$ & 0.9 \\
Plank's Constant & $\tilde{h}_{p}$ & $6.6 \times 10^{-34} \mathrm{~m}^{2} \mathrm{Kg} / \mathrm{s}$ \\
Wavelength & $\lambda$ & $1550 \mathrm{~nm}$ \\
Receiver Load & $R_{l}$ & $100 \Omega$ \\
Receiver Temperature & $T_{r}$ & $300^{\circ} \mathrm{K}$ \\
Channel Loss & $h_{l}$ & $30 \mathrm{~dB}$ \\
Background Noise Power & $P_{b}$ & $10 \mathrm{nW}$ \\
\hline Weak turbulence & $\sigma_{R}^{2}$ & 0.4 \\
channel parameters & $\alpha$ & 6.9 \\
& $\beta$ & 5.3 \\
\hline Strong turbulence & $\sigma_{R}^{2}$ & 2 \\
channel parameters & $\alpha$ & 3.9 \\
& $\beta$ & 1.7 \\
\hline \hline
\end{tabular}

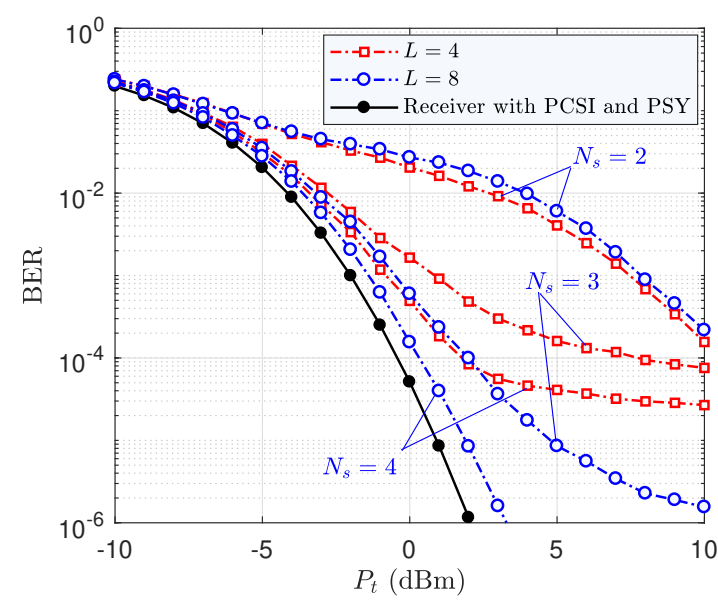

Fig. 4. BER of ML-based detection method for for $\sigma_{R}^{2}=0.4$ and different values of $L$ and $N_{s}$, compared to the case of PCSI and PSY.

\section{Simulation Results}

We present here a set of numerical results to study the system performance for the proposed detection methods. The main considered parameters for the simulation results are summarized in Table I, mostly adopted from recent literature, e.g. [13], [35]. Unless otherwise specified, we consider the case of the weak turbulence regime. Link performance is mainly evaluated in terms of the average bit-error-rate (BER) where the case of the Rx with PCSI and PSY is considered as a lower bound benchmark.

To investigate the impact of the number of samplers and the observation window length on the performance of the first (ML-based) proposed detection method, we have presented in Fig. 4 BER plots versus $P_{t}$ for different values of $N_{s}$ and $L$, assuming that $f_{h}(h)$ is known at the Rx. As expected, the link performance improves by increasing $N_{s}$ and $L$, with a strong dependence on the former; the improvement is especially

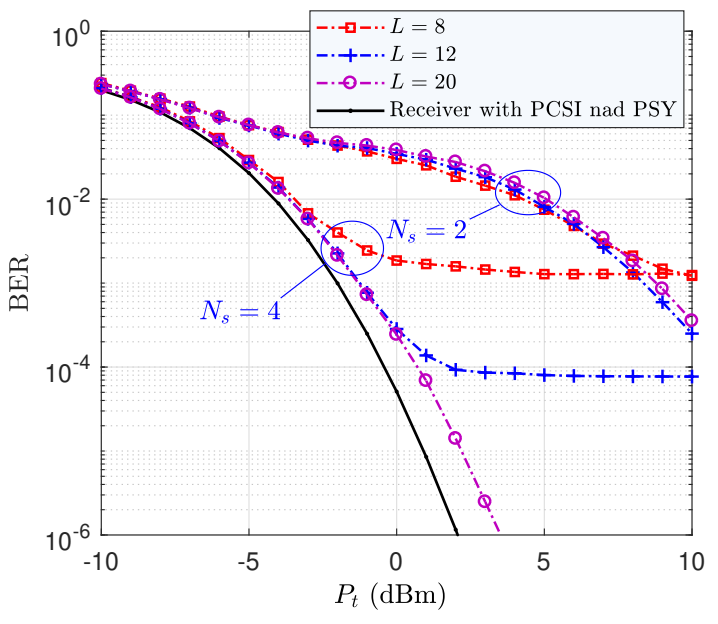

Fig. 5. BER of GLRT-based detector for $\sigma_{R}^{2}=0.4$ and different values of $L$ and $N_{s}$, compared to the case of PCSI and PSY.

significant when $N_{s}$ increases from two to three. In fact, for relatively small $N_{s}$, the system performance is limited by inter-symbol interference resulting from synchronization errors. As $N_{s}$ increases, the standard deviation of timing jitter decreases, and hence, the resulting inter symbol interference. From these results, we can suggest $L=8$ and $N_{s}=4$ as suitable choices for the proposed detector, where we can achieve a performance close to the PSCI and PSY case; e.g. with a gap of smaller than $2 \mathrm{~dB}$ at a target BER of $10^{-6}$. Let us now investigate the performance of the second (GLRTbased) proposed detector. We have presented in Fig. 5 the BER plots, where as expected, by increasing $N_{s}$ and $L$, the BER approaches that of the Rx with PCSI and PSY. Here, $N_{s}=4$ and $L=20$ appear to be a suitable choice. It is worth mentioning that this larger $L$ does not mean a higher computational complexity, compared with the ML-based one (for which $L=8$ was a suitable choice, see above). To clarify this, for $L=8$ and $N_{s}=4$, to detect a sequence of 8 bits, for instance, the ML-based detector requires to compute $2^{8+1}$ metrics of (13), involving the calculation of an two-dimensional integral, while in addition, requiring the knowledge of $f_{h}(h)$. The GLRT-based detector, on the other hand, needs to compute $2 \times 4 \times(20+1)$ metrics of (26) for $N_{s}=4$ and $L=20$.

\section{Sequence Detection of Differential OOK MODULATION}

We consider now the case of employing differential OOK signaling (see Subsection II-B and Fig. 3) in two special cases where the corresponding channel fading coefficients have very high or otherwise very low correlation. These correspond to the cases where $\lambda_{1}$ and $\lambda_{2}$ are selected as very close or very different, respectively. The former is suitable when working in relatively weak turbulence, whereas the latter offers diversity benefit under relatively strong turbulence conditions.

Before describing the blind detection methods, we present in the following the BER calculation for the case of PCSI (i.e., perfectly known $h_{1}$ and $h_{2}$ ) and PSY (i.e., $\tau_{i}=0$ ) at the Rx. 
For PSY receiver, (6) is simplified as

$$
r_{d, k}=\eta\left(h_{1} s_{k}-h_{2} \bar{s}_{k}\right)+n_{d, k},
$$

where $n_{d, k}$ is a zero mean Gaussian noise with variance $\sigma_{d, n}^{2}=\sigma_{s}^{2} h_{1} s_{k}+\sigma_{s}^{2} h_{2} \bar{s}_{k}+\sigma_{0}^{2}$.

\section{A. BER Analysis Under PCSI and PSY}

For the case of highly correlated $h_{1}$ and $h_{2}$, i.e., $h_{1} \approx h_{2}$, the average BER, denoted by $P_{e}$ is given by

$$
P_{e}=\int_{0}^{\infty} P_{e \mid h}(h) f_{h}(h) d h,
$$

where

$$
\begin{aligned}
P_{e \mid h}(h) & =\frac{1}{2} \operatorname{Prob}\left\{r_{d, k}<\gamma_{t h} \mid s_{k}=1\right\} \\
& +\frac{1}{2} \operatorname{Prob}\left\{r_{d, k}>\gamma_{t h} \mid s_{k}=0\right\} \\
& =\frac{1}{2} Q\left(\frac{\gamma_{\mathrm{th}}+\eta h}{\sqrt{\sigma_{s}^{2} h+\sigma_{0}^{2}}}\right)+\frac{1}{2} Q\left(\frac{\eta h-\gamma_{\mathrm{th}}}{\sqrt{\sigma_{s}^{2} h+\sigma_{0}^{2}}}\right)
\end{aligned}
$$

and $Q($.$) is the well known Q$-function and $\gamma_{\text {th }}$ denotes the detection threshold. By differentiating (30) and setting the result to zero, the optimal detection threshold is obtained as $\gamma_{\text {th,opt }}=0$, as expected. Then, (29) is simplified as

$$
P_{e}=\int_{0}^{\infty} Q\left(\frac{\eta h}{\sqrt{\sigma_{s}^{2} h+\sigma_{0}^{2}}}\right) f_{h}(h) d h .
$$

For the case of independent $h_{1}$ and $h_{2}$, we have:

$$
P_{e}=\int_{0}^{\infty} \int_{0}^{\infty} P_{e \mid h_{1}, h_{2}}\left(h_{1}, h_{2}\right) f_{h_{1}}\left(h_{1}\right) f_{h_{2}}\left(h_{2}\right) d h_{1} d h_{2} .
$$

Based on (6), we can write:

$$
\begin{aligned}
& P_{e \mid h_{1}, h_{2}}\left(h_{1}, h_{2}\right)= \\
& \quad \frac{1}{2} Q\left(\frac{\gamma_{\mathrm{th}}+\eta h_{2}}{\sqrt{\sigma_{s}^{2} h_{2}+\sigma_{0}^{2}}}\right)+\frac{1}{2} Q\left(\frac{\eta h_{1}-\gamma_{\mathrm{th}}}{\sqrt{\sigma_{s}^{2} h_{1}+\sigma_{0}^{2}}}\right) .
\end{aligned}
$$

To obtain the optimal detection threshold, by differentiating (33) and setting the result to zero, we get:

$$
\begin{aligned}
& \gamma_{\text {th }, \text { opt }}=-\frac{\eta h_{2}\left(\sigma_{s}^{2} h_{1}+\sigma_{0}^{2}\right)+\eta h_{1}\left(\sigma_{s}^{2} h_{2}+\sigma_{0}^{2}\right)}{\sigma_{s}^{2}\left(h_{1}-h_{2}\right)} \\
& +\frac{1}{\sigma_{s}^{2}\left(h_{1}-h_{2}\right)}\left[\left(\eta h_{2}\left(\sigma_{s}^{2} h_{1}+\sigma_{0}^{2}\right)+\eta h_{1}\left(\sigma_{s}^{2} h_{2}+\sigma_{0}^{2}\right)\right)^{2}\right. \\
& -\sigma_{s}^{2}\left(h_{1}-h_{2}\right)\left(\eta^{2} h_{2}^{2}\left(\sigma_{s}^{2} h_{1}+\sigma_{0}^{2}\right)-\eta^{2} h_{1}^{2}\left(\sigma_{s}^{2} h_{2}+\sigma_{0}^{2}\right)\right. \\
& \left.\left.-\left(\sigma_{s}^{2} h_{1}+\sigma_{0}^{2}\right)\left(\sigma_{s}^{2} h_{2}+\sigma_{0}^{2}\right) \ln \left(\frac{\sigma_{s}^{2} h_{1}+\sigma_{0}^{2}}{\sigma_{s}^{2} h_{2}+\sigma_{0}^{2}}\right)\right)\right]
\end{aligned}
$$

From (7), in differential signaling case, for $h_{1}=h_{2}=h$, the mean of received signal from bits ' 0 ' and ' 1 ' are $-\eta h$ and $\eta h$, respectively, and have the same variances. Hence, for differential signaling with $h_{1}=h_{2}$, due to the symmetry of the received signals around zero, when $h_{1}$ tends to $h_{2}, \gamma_{\text {th,opt }} \rightarrow 0$, as expected. Also, for the case of signal-independent noise

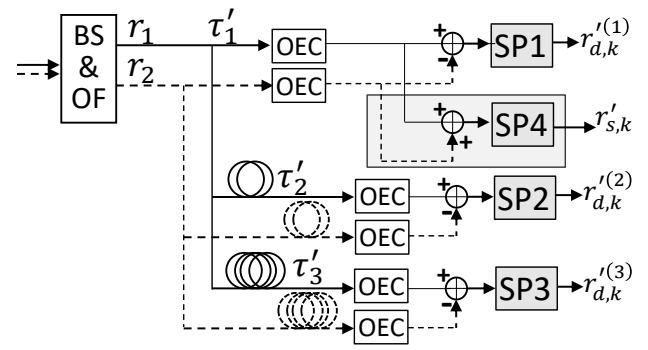

Fig. 6. Simplified Rx block diagram from Fig. 3 for differential signaling with highly correlated $h_{1}$ and $h_{2}$.

at the $\mathrm{Rx}$, this optimal value reduces to $\left(h_{1}+h_{2}\right) / 2$, as considered in [25], [36].

In the sequel, we propose blind signal detection methods for the case of differential signaling. We consider signal detection based on the GLRT criterion due to the high computationally complexity of the ML detector, as explained in the previous section for the case of single-wavelength transmission.

\section{B. Differential Sequence Detection Method for the Case of Highly-Correlated Channels}

Consider first the case where $\lambda_{1}$ and $\lambda_{2}$ are very close, resulting in $h_{1} \approx h_{2}=h$, with the benefit of suppressing the effect of background noise [21]. For this case, the block diagram of the Rx structure in Fig. 3 is simplified to that given in Fig. 6 (the block $\mathrm{SP}_{4}$ will be explained later). Here, (6) can be simplified as

$$
r_{d, k}^{(i)}=\eta h\left(1-\tau_{i}\right) s_{d, k}+\eta h \tau_{i} s_{d, k+1}+n_{d, k}^{(i)}
$$

where $s_{d, k}=s_{k, 1}-s_{k, 2} \in\{-1,1\}$. In this case, unlike $n_{k}^{(i)}$ in (3), the variance of $n_{d, k}^{(i)}$ is independent from both the transmitted symbols and $\tau_{i}$. As a result, we have

$$
\sigma_{d, n}^{2}=\sigma_{s, n}^{2}=\sigma_{s}^{2} h+\sigma_{d, 0}^{2}, \text { with } \sigma_{d, 0}^{2}=2 \sigma_{0}^{2} .
$$

Using (35), we can write

$$
\begin{aligned}
& p\left[r_{d, k}^{(i)} \mid h, \tau_{i}, s_{k}, s_{k+1}\right]=\frac{1}{\sqrt{2 \pi\left(\sigma_{s}^{2} h+\sigma_{d, 0}^{2}\right)}} \\
& \quad \times \exp \left(-\frac{\left|r_{d, k}^{(i)}-\eta h\left(1-\tau_{i}\right) s_{d, k}-\eta h \tau_{i} s_{d, k+1}\right|^{2}}{2\left(\sigma_{s}^{2} h+\sigma_{d, 0}^{2}\right)}\right) .
\end{aligned}
$$

Let $\mathbf{r}_{d, i}(L)=\left\{r_{d, 1}^{(i)}, r_{d, 2}^{(i)}, \ldots, r_{d, L}^{(i)}\right\}$ denote the output sequence of $\mathrm{SP}_{\mathrm{i}}$ sampler. Using (37), and assuming $h$ is known at the $\mathrm{Rx}$, the GLRT detection (see (16)) gives:

$$
\hat{i}, \hat{\mathbf{s}}(L)=\underset{i, \mathbf{s}(L+1)}{\arg \max } p\left[\mathbf{r}_{d, i}(L) \mid h, \tau_{i} \in\left[0,1 / N_{s}\right), \mathbf{s}(L+1)\right],
$$


where

$$
\begin{aligned}
& p\left[\mathbf{r}_{d, i}(L) \mid h, \tau_{i} \in\left[0,1 / N_{s}\right), \mathbf{s}(L+1)\right] \\
& =N_{s} \int_{0}^{\frac{1}{N_{s}}} p\left[\mathbf{r}_{d, i}(L) \mid h, \tau_{i}, \mathbf{s}(L+1)\right] d \tau_{i} \\
& =\frac{\mathcal{D}_{1}^{\prime}}{\sqrt{\mathcal{D}_{3}^{\prime}}} Q\left(\frac{N_{s} \sqrt{\mathcal{D}_{3}^{\prime}}}{\sqrt{\sigma_{s}^{2} h+\sigma_{d, 0}^{2}}}-\frac{\mathcal{D}_{2}^{\prime}}{\mathcal{D}_{3}^{\prime}}\right) \\
& \left\{\begin{aligned}
\mathcal{D}_{1}^{\prime}= & \frac{N_{s} \exp \left(\frac{\mathcal{D}_{2}^{\prime 2} / \mathcal{D}_{3}^{\prime}-\sum_{k=1}^{L}\left(r_{d, k}^{(i)}-\eta h s_{d, k}\right)^{2}}{2\left(\sigma_{s}^{2} h+\sigma_{d, 0}^{2}\right)}\right)}{\left(2 \pi\left(\sigma_{s}^{2} h+\sigma_{d, 0}^{2}\right)\right)^{L / 2-1}} \\
\mathcal{D}_{2}^{\prime}= & \eta h \sum_{k=1}^{L}\left(r_{d, k}^{(i)}-\eta h s_{d, k}\right) s_{d, k}^{\prime \prime} \\
\mathcal{D}_{3}^{\prime}= & \eta^{2} h^{2} \sum_{k=1}^{L} s_{d, k}^{\prime \prime 2}
\end{aligned}\right.
\end{aligned}
$$

and $s_{d, k}^{\prime \prime}=s_{d, k+1}-s_{d, k}$.

Since $s_{k}+\bar{s}_{k}=1$, in order to estimate $h$, we pass $r_{1}+r_{2}$ through an additional sampler, e.g. $\mathrm{SP}_{4}$ as shown in Fig. 6. At the output of this latter, we have:

$$
r_{s, k}^{\prime(i)}=\eta h+n_{s, k},
$$

where $n_{s, k}$ is an additive Gaussian noise with zero mean and variance $\sigma_{s}^{2} h+2 \sigma_{0}^{2}$. Now, using (41), we can obtain an ML estimate of $h$ as

$$
\hat{h}=\frac{1}{\eta L} \sum_{k=1}^{L} r_{s, k}^{(i)}=h+n_{h}^{(i)}
$$

where $n_{h}^{(i)}=\frac{1}{\eta L} \sum_{k=1}^{L} n_{s, k}^{\prime(i)}$ is a Gaussian noise with zero mean and variance $\sigma_{n, h}^{2}=\left(\sigma_{s}^{2} h+2 \sigma_{0}^{2}\right) / \eta^{2} L$. So, we can easily estimate $h$ even without any information on $s_{k}$ and $\tau_{i}$. By substituting $h$ by $\hat{h}$ in (39), $\mathbf{s}(L)$ detection is done by searching over $N_{s} \times 2^{L+1}$ possible states of metric (39). It is worth mentioning that, unlike metric (13), (39) consists of a closed-form expression.

Note that, to detect data from (38), the detector requires to calculate and compare $N_{s} \times 2^{L+1}$ possible states of the metric (39) which increases exponentially by increasing $L$. The proposed detection method of (38) can be considerably simplified in the high SNR regime where the effect of noise can be ignored compared with the sampling clock offset. Remember that we should find which $\tau_{i}, i \in\left\{1, \ldots, N_{s}\right\}$, gets its value in the interval $\left[0,1 / N_{s}\right)$. For a given $i$, if $s_{k}=1, r_{d, k}^{(i)}$ takes the positive values $\eta h$ or $\eta\left(1-\tau_{i}\right)$. Likewise, if $s_{k}=0$, $r_{d, k}^{(i)}$ takes the negative values $-\eta h$ or $-\eta\left(1-\tau_{i}\right)$. Now, for the specific $i$ where $\tau_{i}$ falls in the interval $\left[0,1 / N_{s}\right)$, we have with a high probability $r_{d, k}^{(i)}>0$ for $s_{k}=1$ and $r_{d, k}^{(i)}<0$ otherwise. Using this and following the method developed in Appendix, it can be shown that the metric (38) is maximized when $N_{d}$ positive values of $\mathbf{r}_{d, i}(L)$ are chosen as bit " 1 " and $L-N_{d}$ negative values as bit " 0 ". Hence, the search space is reduced from $N_{s} \times 2^{L+1}$ to $2 N_{s}$ where the factor 2 is related to the two possible states ( 0 or 1 ) of $s_{L+1}$.

\section{Differential Sequence Detection Method for the Case of Independent Channels}

Consider now the special case where $\lambda_{1}$ and $\lambda_{2}$ are sufficiently different so that we can assume almost independent $h_{1}$ and $h_{2}$. Under such conditions, we can potentially benefit from a diversity gain [25], which makes this scheme interesting for moderate to strong turbulence regimes. However, we need almost twice number of samplers, compared to the correlated case (see Figs. 3 and 6), resulting in a more complex Rx. Here, $p\left[r_{d, k}^{(i)} \mid h_{1}, h_{2}, \tau_{i}, s_{k}, s_{k+1}\right]$ can be expressed as in (43) on the top of the next page. Notice that in contrast to the correlated scenario, here we have to detect the received signals in the presence of three unknown parameters, i.e., $h_{1}, h_{2}$, and $\tau$. For this, we propose a two-step detection method as described in the following.

1) First Step: An initial estimate for $h_{1}$ and $h_{2}$ is obtained as [37]

$$
\hat{h}_{m}=\eta^{-1} \max \left\{r_{1, m}^{(i)}, r_{2, m}^{(i)}, \ldots, r_{L, m}^{(i)}\right\}, \text { for } m \in\{1,2\} .
$$

Now, similar to the GLRT methodology, by substituting (6) and (44) in (20), the first decision step is expressed as

$$
\hat{m}, \hat{i}, \hat{\mathbf{s}}(L)=\underset{m, i, \mathbf{s}(L+1)}{\arg \min } \mathcal{A}_{2},
$$

where

$$
\mathcal{A}_{2}=\sum_{k=1}^{L} \frac{\left|r_{k, m}^{(i)}-\eta \hat{h}_{m} s_{k, m}^{\prime}\right|^{2}}{\hat{h}_{m} s_{k, m}^{\prime} \sigma_{s}^{2}+\sigma_{0}^{2}}+\log \left(\hat{h}_{m} s_{k, m}^{\prime} \sigma_{s}^{2}+\sigma_{0}^{2}\right),
$$

$m \in\{1,2\}, s_{k, 1}=s_{k}, s_{k, 2}=\bar{s}_{k}$, and

$$
s_{k, m}^{\prime}=\left(1-\frac{1}{2 N_{s}}\right) s_{k, m}+\frac{1}{2 N_{s}} s_{k+1, m} .
$$

Using the same approach we proposed in Appendix to reduce the complexity of calculation of (26), here we can reduce the search space of (45) to $8 N_{s} L$, which is twice that of the GLRT-based method for the case of single wavelength transmission (see Appendix for more details).

2) Second Step: Now, assuming error-free signal detection in the first step, we use ML-based estimation of $h_{m}$ to improve the accuracy of the initial estimate in (45):

$$
\hat{h}_{m}=\frac{\sum_{k=1}^{L-1} r_{k, m}^{(i)}\left(\left(1-\tau_{i}\right) s_{k}+\tau_{i} s_{k+1}\right)}{\eta \sum_{k=1}^{L-1}\left(\left(1-\tau_{i}\right) s_{k}+\tau_{i} s_{k+1}\right)^{2}} .
$$

where we replace $\mathbf{s}(L)=\left\{s_{1}, s_{2}, \ldots, s_{L}\right\}$ by $\hat{\mathbf{s}}(L)$ from the first detection step and $\tau_{i}$ by $\bar{\tau}_{i}=1 / 2 N_{s}$. Using the improved $\hat{h}_{m}$ from (48), and similar to (20), we can formulate the second step of data detection as

$$
\hat{i}, \hat{\mathbf{s}}(L)=\underset{i, \mathbf{s}(L+1)}{\arg \min } \mathcal{A}_{3},
$$




$$
p\left[r_{d, k}^{(i)} \mid h_{1}, h_{2}, \tau_{i}, s_{k}, s_{k+1}\right]=\frac{\exp \left(-\frac{\left|r_{d, k}^{(i)}-\eta\left(1-\tau_{i}\right)\left(h_{1} s_{k}-h_{2} \bar{s}_{k}\right)-\eta \tau_{i}\left(h_{1} s_{k+1}-h_{2} \bar{s}_{k+1}\right)\right|^{2}}{2\left[\left(1-\tau_{i}\right)\left(h_{1} s_{k}+h_{2} \bar{s}_{k}\right)+\tau_{i}\left(h_{1} s_{k+1}+h_{2} \bar{s}_{k+1}\right)\right] \sigma_{s}^{2}+2 \sigma_{0}^{2}}\right)}{\sqrt{2 \pi\left(\left[\left(1-\tau_{i}\right)\left(h_{1} s_{k}+h_{2} \bar{s}_{k}\right)+\tau_{i}\left(h_{1} s_{k+1}+h_{2} \bar{s}_{k+1}\right)\right] \sigma_{s}^{2}+\sigma_{0}^{2}\right)}}
$$

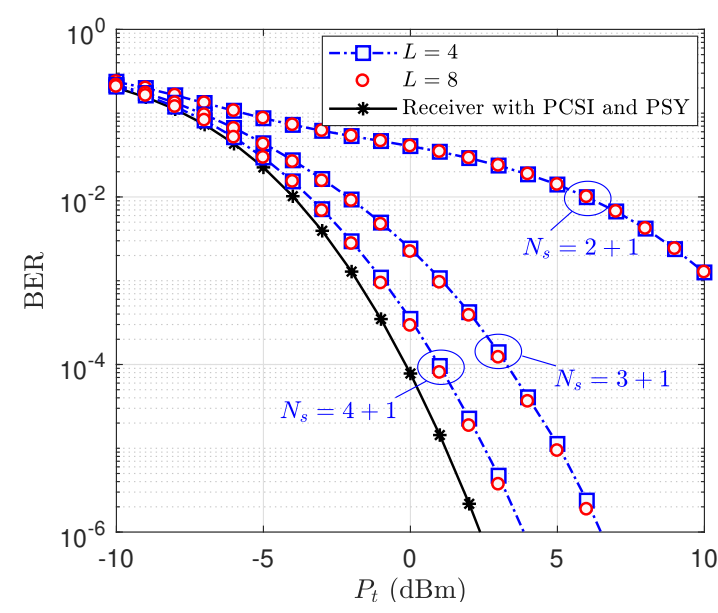

Fig. 7. BER Performance of scheme $\mathrm{C}$ for $\sigma_{R}^{2}=0.4$ and different values of $L$ and $N_{s}$, compared to the BER achieved with PCSI and PSY.

where

$$
\mathcal{A}_{3}=\sum_{k=1}^{L} \log \left(p\left[r_{d, k}^{(i)} \mid \hat{h}_{1}, \hat{h}_{2}, \tau_{i}=\bar{\tau}, s_{k}, s_{k+1}\right]\right) .
$$

As explained in Appendix, the minimum of $\mathcal{A}_{3}$ is obtained when $N_{d}$ positive values of $\mathbf{r}_{d, i}(L)-\gamma_{\mathrm{th}, \mathrm{opt}}$ are detected as bit ' 1 ' and $L-N_{d}$ negative values of $\mathbf{r}_{d, i}(L)-\gamma_{\mathrm{th}, \mathrm{opt}}$ are detected as bit ' 0 ', with $\gamma_{\text {th,opt }}$ given in (34), which is calculated by substituting $h_{1}$ and $h_{2}$ by their estimates from (48). This way, the search space for finding the minimum of $\mathcal{A}_{3}$ is reduced from $N_{s} \times 2^{L+1}$ to $2 N_{s}$. The total search space associated with the first and the second steps is hence $2 N_{s}(1+4 L)$, which is much smaller than the initial search space of $2^{L}$.

\section{Simulation Results}

To study the performance of the proposed blind detectors, consider first the case of highly correlated channels. In Fig. 7, we have presented the BER plots for different values of $N_{s}$ and $L$. Here, $L=4$ and $N_{s}=5$ can be considered as appropriate choices since the obtained performance is quite close to the detector with PSY and PCSI, with an SNR gap of less than $2 \mathrm{~dB}$ at a target BER of $10^{-6}$. This requirement to relatively small $N_{s}$ and $L$ makes this scheme interesting from a computational complexity point of view. Let us now investigate the case of independent $h_{1}$ and $h_{2}$. We focus on the strong turbulence regime where having independent $h_{1}$ and $h_{2}$ allows to benefit from a diversity gain here. We have contrasted in Fig. 8 the BER performance of the proposed detector for different $L$ and $N_{s}$. For the sake of presentation clarity, the cases of $N_{s}=4,6$, and 8, are presented separately, where we again notice the significant impact of $N_{s}$ on the Rx performance. For instance, from Fig. 8a, for a relatively small value of $N_{s}=4$ (i.e., using two samplers on each wavelength), we do not obtain any performance improvement by increasing $L$ and/or $P_{t}$. From Fig. 8 for $N_{s}=8$ and $L=16$, a BER performance close to the ideal receiver is achieved, with an SNR gap of less than $2 \mathrm{~dB}$ at a target BER of $10^{-6}$. The requirement to larger $N_{s}$ and $L$, compared with the correlated case, and the resulting higher computational complexity can be justified for the strong turbulence regime by the significant performance improvement obtained due to the link diversity.

\section{E. Complexity Comparison}

Besides the presented simulation results, in order to draw general conclusions on the advantage of the proposed blind detectors, we have compared them from an implementation complexity point of view in Table II.

\section{CONCLUSion ANd Future Work}

In this work, we investigated optimal signal detection for an FSO link with high mobility under practical conditions where the Rx does not have any knowledge about the instantaneous channel fading coefficients and, in addition, it experiences sampling clock offset. The main idea behind the proposed schemes was to use multiple samplers at the Rx and to perform blind sequence detection on the resulting signal samples. First, we proposed two sequence data detectors based on ML and GLRT criteria for conventional OOK signaling where a single wavelength is used for signal transmission. Due to the relatively high computational complexity of these detectors, in a second step, we proposed a differential signaling structure based on the transmission of two wavelengths that can be selected as too close or otherwise very different. The former scheme is interesting under weak turbulence conditions, allowing to suppress the background noise effect, whereas the latter offers link diversity and, as a result, a significant performance improvement under relatively strong turbulence conditions. We proposed efficient blind signal detection methods for these two schemes and illustrated their efficiency through numerical results. Also, we discussed and contrasted the computational complexity of the proposed detectors, in particular, regarding the choice of the number of samplers and the frame length. It is worth mentioning that the suitable choice of these parameters does not depend on channel conditions.

In our work we assumed that the mobile transceiver are perfectly aligned. One way to align the mobile transceiver is by using an array of photodetector at the receiver to track the transmitter beam which may be associated with error. Future work will focus on studying and designing the receiver that 


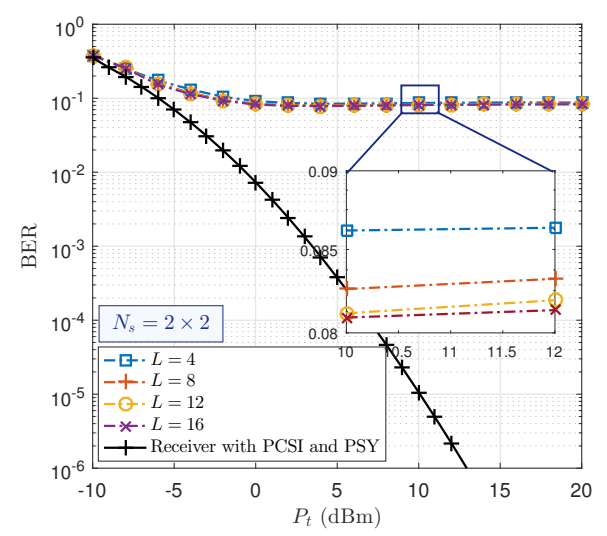

(a)

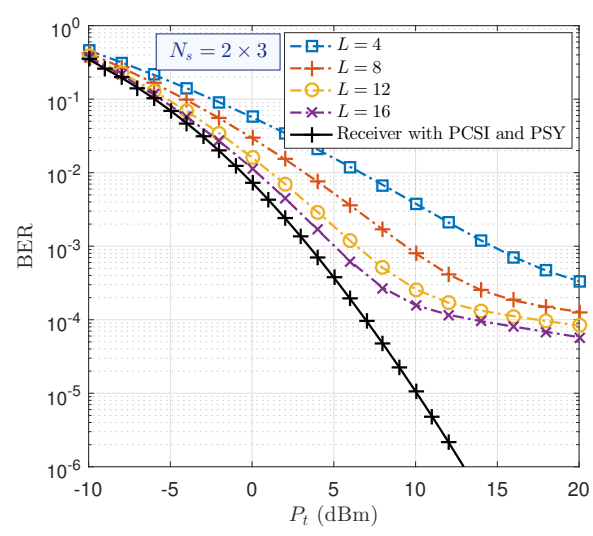

(b)

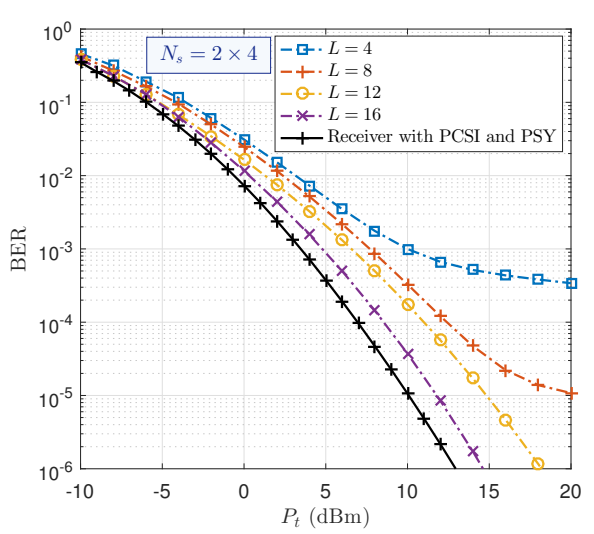

(c)

Fig. 8. BER Performance of scheme D for $\sigma_{R}^{2}=2$ and different values of $L$ and $N_{s}$, compared to the BER achieved with PCSI and PSY for: (a) $N_{s}=2 \times 2=4 ;$ (b) $N_{s}=2 \times 3=6 ;$ (c) $N_{s}=2 \times 4=8$.

TABLE II

COMPUTATIONAL COMPLEXITY COMPARISON OF PROPOSED DATA DETECTION SCHEMES.

\begin{tabular}{|c|c|c|c|c|c|}
\hline Methods & $\begin{array}{c}\text { Required } \\
N_{s} \\
\end{array}$ & $\begin{array}{c}\text { Required } \\
L_{s} \\
\end{array}$ & $\begin{array}{c}\text { Search } \\
\text { Space }\end{array}$ & Comments & $\begin{array}{c}\text { Computational } \\
\text { Complexity }\end{array}$ \\
\hline $\begin{array}{c}\text { ML }, \\
\text { Single } \\
\text { Wavelength }\end{array}$ & 4 & 8 & $\begin{array}{l}2^{L+1} \\
=512\end{array}$ & $\begin{array}{c}\text { ML-based detector, conventional OOK signaling; requires to know } \\
f_{h}(h) \text { and solving two-dimensional integrations for each metric. } \\
\text { Search space is too large. }\end{array}$ & Very high \\
\hline $\begin{array}{l}\text { GLRT, } \\
\text { Single } \\
\text { Wavelength }\end{array}$ & 4 & 20 & $\begin{array}{l}4 N_{S} L \\
=320\end{array}$ & $\begin{array}{c}\text { GLRT-based detector, conventional OOK signaling; does not require to know } \\
f_{h}(h) \text {; each metric consists of a series of additions and } \\
\text { multiplications. Search space is relatively large. }\end{array}$ & Medium \\
\hline $\begin{array}{l}\text { Differential } \\
\text { Signaling, } \\
\text { Correlated } \\
\text { Channels }\end{array}$ & $\begin{array}{l}4+1 \\
=5\end{array}$ & 4 & $\begin{array}{c}2 N_{S} \\
=8\end{array}$ & $\begin{array}{l}\text { GLRT-based detector, differential OOK signaling; does not require to know } \\
f_{h}(h) \text {; each metric consists of a series of additions and } \\
\text { multiplications. Search space is very small. Closed-form formulation } \\
\text { was derived for the metric. Suitable for weak turbulence conditions. }\end{array}$ & Low \\
\hline $\begin{array}{l}\text { Differential } \\
\text { Signaling, } \\
\text { Independent } \\
\text { Channels }\end{array}$ & $\begin{array}{c}2 \times 4 \\
=8\end{array}$ & 16 & $\begin{array}{c}2 N_{s}(4 L+1) \\
=1040\end{array}$ & $\begin{array}{l}\text { GLRT-based detector, differential OOK signaling; does not require to know } \\
f_{h}(h) \text {; each metric calculation consists of a series of additions and multiplications. } \\
\text { Search space is relatively large. } \\
\text { Suitable for strong turbulence conditions. }\end{array}$ & $\begin{array}{c}\text { Relatively } \\
\text { high }\end{array}$ \\
\hline
\end{tabular}

jointly synchronize and track the received optical signal before data detection.

\section{APPENDIX}

To avoid the exhaustive search in the GLRT detector, we develop here a fast search algorithm to implement (25). Let us show that for a fixed $N_{1}=\sum_{k=1}^{L-1} s_{k}$, the minimum value of metric $\mathcal{A}_{1}$ is obtained when $\mathcal{R}_{o n, 1}=\sum_{k=1}^{L-1} r_{k}^{(i)} s_{k}$ takes its maximum value. The maximum value of $\mathcal{R}_{o n, 1}$ is achieved when $N_{1}$ observations among $L-1$ observations $\mathbf{r}_{i}(L-1)=\left\{r_{1}^{(i)}, \ldots, r_{L-1}^{(i)}\right\}$ are detected as bit one. As $N_{1} \in\{0,1, \ldots, L-1\}$ takes $L$ different values, the search space is reduced from $N_{s} \times 2^{L+1}$ possible states to $4 N_{s} L$, where $N_{s}$ stands for the number of samplers, coefficient 4 characterizes the four possible states of $\left\{s_{L}, s_{L+1}\right\}$ and $L$ stands for the number of possible states of $N_{1}$.

In the sequel, we prove that the metric $\mathcal{A}_{1}$ is a decreasing function of $\mathcal{R}_{o n, 1}$. According to (27), in the case of signal independent noise, it is obvious that $\mathcal{A}_{1}$ is a decreasing function of $\mathcal{R}_{o n, 1}$. So, we consider the general shot noise and thermal noise scenario characterized by (26). We consider the worst case where $N_{1}<L / 2$ and for $k \in\{1,2, \ldots, L-1\}$ if $s_{k}=1$, then $s_{k+1}$ and $s_{k-1}$ are equal to zero. In other words, the worst case here is when the received sequence does not contain two consecutive bits "1". In this worst case, $\mathcal{A}_{1}$ can be rewritten as

$$
\begin{aligned}
\mathcal{A}_{1}= & -\frac{2 \eta}{\sigma_{s}^{2}} \underbrace{\left(\mathcal{B}_{1} \mathcal{R}_{o n, 1}^{(i)}+\mathcal{B}_{2} \mathcal{R}_{o n, 1}^{\prime(i)}\right)}_{I_{1}}+N_{1} \log \mathcal{B}_{1}^{\prime} \\
& -\frac{1}{\sigma_{0}^{2}} \underbrace{\left(\mathcal{B}_{1} \mathcal{R}_{o n, 2}^{(i)}+\mathcal{B}_{2} \mathcal{R}_{o n, 2}^{\prime(i)}\right)}_{I_{2}}+N_{1} \log \mathcal{B}_{2}^{\prime}+\mathrm{C}_{1},
\end{aligned}
$$

where $\mathcal{B}_{1}=\frac{\mathcal{B}_{1}^{\prime}}{\mathcal{B}_{1}^{\prime}+\sigma_{0}^{2}}, \mathcal{B}_{2}=\frac{\mathcal{B}_{2}^{\prime}}{\overline{\mathcal{B}_{2}^{\prime}+\sigma_{0}^{2}}}, \mathcal{B}_{1}^{\prime}=\hat{h} \sigma_{s}^{2}\left(1-\frac{1}{2 N_{s}}\right)$, $\mathcal{B}_{2}^{\prime}=\hat{h} \sigma_{s}^{2} \frac{1}{2 N_{s}}$, and $\mathrm{C}_{1}$ is independent from both $\mathbf{s}^{\prime}(L-1)$ and $\mathbf{r}_{i}(L-1)$. Note that, $y=x /(x+a)$ is an increasing function of $x$ where $a$ is a constant term. As $\hat{h}$ is an increasing function of $\mathcal{R}_{o n, 1}^{(i)}, \mathcal{B}_{1}^{\prime}$ and $\mathcal{B}_{2}^{\prime}$ and consequently $\mathcal{B}_{1}$ and $\mathcal{B}_{2}$ are an increasing function of $\mathcal{R}_{o n, 1}^{(i)}$. Considering the relationships between $\mathcal{R}_{o n, 1}^{(i)}, \mathcal{R}_{o n, 1}^{\prime(i)}, \mathcal{R}_{o n, 2}^{(i)}$ and $\mathcal{R}_{o n, 2}^{\prime(i)}$, it is easily verified that the terms $I_{1}$ and $I_{2}$ increase by increasing $\mathcal{R}_{o n, 1}^{(i)}$. On the other hand, at high SNR regime, we have $\mathbb{E}\left[\mathcal{R}_{o n, 1}^{(i)}\right] / \sigma_{s}^{2} \gg$ 1 and $\mathbb{E}\left[\mathcal{R}_{o n, 2}^{(i)}\right] / \sigma_{0}^{2} \gg 1$, where $\mathbb{E}[$.$] denotes expectation.$ So, we have $\frac{2 \eta}{\sigma_{s}^{2}} I_{1} \gg N_{1} \log \mathcal{B}_{1}^{\prime}$ and $\frac{I_{2}}{\sigma_{0}^{2}} \gg N_{1} \log \mathcal{B}_{2}^{\prime}$. This confirms that for the worst case, the metric $\mathcal{A}_{1}$ is a decreasing function of $\mathcal{R}_{o n, 1}^{(i)}$. Hence, for a given $N_{1}$, instead of searching 
over $N_{s} \times\left(\begin{array}{c}L-1 \\ N_{1}\end{array}\right)$ possible states, we can compute metric $\mathcal{A}_{1}$ for $N_{s}$ cases in which the $N_{1}$ maximum values of $\mathbf{r}_{i}(L-1)$ s are considered as bit " 1 ". This proves that the total search space is reduced from $N_{s} \times 2^{L+1}$ to $4 N_{s} L$. For instance, for $N_{s}=3$ and $L=10$ and 20, the fast search method reduces the search space from 6144 and 6291456 to 120 and 240, respectively.

\section{REFERENCES}

[1] M. T. Dabiri, S. M. S. Sadough, and M. A. Khalighi, "FSO Communication for High Speed Trains: Blind Data Detection and Channel Estimation," in 2018 11th International Symposium on Communication Systems, Networks \& Digital Signal Processing (CSNDSP). IEEE, Jul. 2018, pp. 1-4.

[2] M. A. Khalighi and M. Uysal, "Survey on Free Space Optical Communication: A Communication Theory Perspective," IEEE Commun. Surveys Tuts., vol. 16, no. 4, pp. 2231-2258, Nov. 2014.

[3] Z. Ghassemlooy, W. Popoola, and S. Rajbhandari, Optical wireless communications. CRC Press Boca Raton, FL, Aug. 2012.

[4] S. Sadough and M. A. Khalighi, Radio Communication. IN-TECH Press, 2010, ch. Recent Developments in Channel Estimation and Detection for MIMO Systems,, pp. 337-372.

[5] F. Xu, M. A. Khalighi, P. Caussé, and S. Bourennane, "Channel coding and time-diversity for optical wireless links," Optics Express, vol. 7, no. 2, p. 872887 , Jan. 2009.

[6] X. Zhu and J. M. Kahn, "Free-space optical communication through atmospheric turbulence channels," IEEE Trans. Commun., vol. 50, no. 8 , pp. 1293-1300, 2002.

[7] M. L. Riediger, R. Schober, and L. Lampe, "Fast multiple-symbol detection for free-space optical communications," IEEE Trans. Commun., vol. 57, no. 4, pp. 1119-1128, 2009.

[8] T. Song and P.-Y. Kam, "A robust GLRT receiver with implicit channel estimation and automatic threshold adjustment for the free space optical channel with IM/DD," J. Lightw. Technol., vol. 32, no. 3, pp. 369-383, 2014.

[9] — - "Robust data detection for the photon-counting free-space optical system with implicit CSI acquisition and background radiation compensation," J. Lightw. Technol., vol. 34, no. 4, pp. 1120-1132, 2016.

[10] M. M. Abadi, Z. Ghassemlooy, S. Zvanovec, M. R. Bhatnagar, M.-A. Khalighi, and Y. Wu, "Impact of link parameters and channel correlation on the performance of fso systems with the differential signaling technique," Journal of Optical Communications and Networking, vol. 9, no. 2, pp. 138-148, 2017.

[11] L. Yang, B. Zhu, J. Cheng, and J. F. Holzman, "Free-space optical communications using on-off keying and source information transformation," J. Lightw. Technol., vol. 34, no. 11, pp. 2601-2609, 2016.

[12] S. Ding, J. Zhang, and A. Dang, "Adaptive threshold decision for on-off keying transmission systems in atmospheric turbulence," Optics express, vol. 25, no. 20, pp. 24 425-24 436, 2017.

[13] M. T. Dabiri, S. M. S. Sadough, and M. A. Khalighi, "FSO channel estimation for OOK modulation with APD receiver over atmospheric turbulence and pointing errors," Optics Communications, vol. 402, pp. 577-584, Nov. 2017.

[14] M. T. Dabiri and S. M. S. Sadough, "Performance analysis of EM-based blind detection for ON-OFF keying modulation over atmospheric optical channels," Optics Communications, vol. 413, pp. 299-303, Apr. 2018.

[15] M. T. Dabiri, S. M. S. Sadough, and H. Safi, "GLRT-based sequence detection of OOK modulation over FSO turbulence channels," IEEE Photon. Technol. Lett., vol. PP, no. 99, pp. 1-1, 2017.

[16] M. T. Dabiri and S. M. S. Sadough, "Generalized blind detection of OOK modulation for free-space optical communication," IEEE Commun. Lett., vol. 21, no. 10, pp. 2170-2173, Oct. 2017.

[17] A. Sniady and J. Soler, "LTE for railways: Impact on performance of ETCS railway signaling," IEEE Veh. Technol. Mag., vol. 9, no. 2, pp. 69-77, 2014.

[18] S. Fathi-Kazerooni, Y. Kaymak, R. Rojas-Cessa, J. Feng, N. Ansari, M. Zhou, and T. Zhang, "Optimal positioning of ground base stations in free-space optical communications for high-speed trains," IEEE Trans. Intell. Transp. Syst., vol. 19, no. 6, pp. 1940-1949, Jun. 2018.
[19] Q. Fan, M. Taheri, N. Ansari, J. Feng, R. Rojas-Cessa, M. Zhou, and T. Zhang, "Reducing the impact of handovers in ground-to-train free space optical communications," IEEE Trans. Veh. Technol., vol. 67, no. 2, pp. 1292-1301, Feb. 2018.

[20] R. Paudel, X. Tang, and Z. Ghassemlooy, "Laboratory demonstration of fso ground-to-train communications with multiple base stations," in 2016 10th International Symposium on Communication Systems, Networks and Digital Signal Processing (CSNDSP),. IEEE, 2016, pp. 1-6.

[21] M. A. Khalighi, F. Xu, Y. Jaafar, and S. Bourennane, "Double-laser differential signaling for reducing the effect of background radiation in free-space optical systems," J. Opt. Commun. Netw., vol. 3, no. 2, pp. 145-154, Feb. 2011.

[22] M. M. Abadi, Z. Ghassemlooy, M. A. Khalighi, S. Zvanovec, and M. R. Bhatnagar, "FSO detection using differential signaling in outdoor correlated-channels condition," IEEE Photon. Technol. Lett., vol. 28, no. 1, pp. 55-58, Jan. 2016.

[23] M. M. Abadi, Z. Ghassemlooy, S. Zvanovec, M. R. Bhatnagar, M. A. Khalighi, and Y. Wu, "Impact of link parameters and channel correlation on the performance of FSO systems with the differential signaling technique," J. Opt. Commun. Netw., vol. 9, no. 2, pp. 138-148, Feb. 2017.

[24] M. M. Abadi, Z. Ghassemlooy, M. R. Bhatnagar, S. Zvanovec, M. A. Khalighi, and A.-R. Maheri, "Using differential signalling to mitigate pointing errors effect in FSO communication link," in IEEE International Conference on Communications (ICC), 2016 Workshops. IEEE, May. 2016, pp. 145-150.

[25] M. R. Bhatnagar, Z. Ghassemlooy, S. Zvanovec, M. A. Khalighi, and M. M. Abadi, "Quantized feedback-based differential signaling for freespace optical communication system," IEEE Trans. Commun., vol. 64, no. 12 , pp. 5176-5188, Dec. 2016.

[26] F. Xu, M. A. Khalighi, and S. Bourennane, "Impact of different noise sources on the performance of PIN-and APD-based FSO receivers," in COST IC0802 Workshop, IEEE ConTEL Conference. IEEE, Jun. 2011, pp. 211-218.

[27] S. V. Kartalopoulos, Free Space Optical Networks for Ultra-broad Band Services. John Wiley \& Sons, 2011.

[28] S. Jeon and H. Toshiyoshi, "MEMS tracking mirror system for a bidirectional free-space optical link," Applied optics, vol. 56, no. 24, pp. 6720-6727, 2017.

[29] M. Alzenad, M. Z. Shakir, H. Yanikomeroglu, and M.-S. Alouini, "FSO-based vertical backhaul/fronthaul framework for $5 \mathrm{G}+$ wireless networks," IEEE Commun. Mag., vol. 56, no. 1, pp. 218-224, 2018.

[30] T. L. Tidwell, J. T. Gregory, C. H. Chalfant III, F. J. Orlando, and M. Leftwich, "Rapid acquisition, pointing and tracking optical system for free space optical communications," Apr. 17 2012, uS Patent $8,160,452$.

[31] L. C. Andrews and R. L. Phillips, Laser Beam Propagation Through Random Media. SPIE press Bellingham, WA, 2005, vol. 1.

[32] H. Lim and D. Yoon, "Generalized Moment-Based Estimation of Gamma-Gamma Fading Channel Parameters," IEEE Trans. Veh. Technol., vol. 67, no. 1, pp. 809-811, 2018.

[33] D. Warrier and U. Madhow, "Spectrally efficient noncoherent communication," IEEE Trans. Inf. Theory, vol. 48, no. 3, pp. 651-668, 2002.

[34] M. Wu and P. Y. Kam, "Performance analysis and computational complexity comparison of sequence detection receivers with no explicit channel estimation," IEEE Trans. Veh. Technol., vol. 59, no. 5, pp. 2625$2631,2010$.

[35] Z. Ghassemlooy, S. Arnon, M. Uysal, Z. Xu, and J. Cheng, "Emerging optical wireless communications-advances and challenges," IEEE J. Sel. Areas Commun., vol. 33, no. 9, pp. 1738-1749, Sep. 2015.

[36] M. M. Abadi, Z. Ghassemlooy, M.-A. Khalighi, S. Zvanovec, and M. R. Bhatnagar, "FSO detection using differential signaling in outdoor correlated-channels condition," IEEE Photon. Technol. Lett., vol. 28, no. 1 , pp. 55-58, 2016.

[37] L. Yang, X. Song, J. Cheng, and J. F. Holzman, "Free-space optical communications over lognormal fading channels using ook with finite extinction ratios," IEEE Access, vol. 4, pp. 574-584, 2016. 